\title{
Reduced Load Equivalence under Subexponentiality
}

PREDRAG JELENKOVIĆ * and PETAR MOMČILOVIĆ*

\{predrag, petar\}@ee.columbia.edu

Department of Electrical Engineering, Columbia University, New York, NY 10027, USA

BERT ZWART

bert.zwart@inria.fr

INRIA, Projet RAP, BP 105, 78153 Le Chesnay, France

Received 24 October 2002; Revised 16 June 2003

\begin{abstract}
The stationary workload $W_{A+B}^{\phi}$ of a queue with capacity $\phi$ loaded by two independent processes $A$ and $B$ is investigated. When the probability of load deviation in process $A$ decays slower than both in $B$ and $\mathrm{e}^{-\sqrt{x}}$, we show that $W_{A+B}^{\phi}$ is asymptotically equal to the reduced load queue $W_{A}^{\phi-b}$, where $b$ is the mean rate of $B$. Given that this property does not hold when both processes have lighter than $\mathrm{e}^{-\sqrt{x}}$ deviation decay rates, our result establishes the criticality of $\mathrm{e}^{-\sqrt{x}}$ in the functional behavior of the workload distribution. Furthermore, using the same methodology, we show that under an equivalent set of conditions the results on sampling at subexponential times hold.
\end{abstract}

Keywords: large deviations, non-Cramér type conditions, reduced load equivalence, independent sampling, subexponential distributions

\section{Introduction}

Statistical resource sharing provides a mechanism for improving operating efficiency in many areas of business and engineering. This mechanism is particularly useful in communication networks, where resources, such as link transmission capacity and buffer space, are shared among different user sessions. This sharing creates potential workload backlogs that need to be addressed. A baseline model of the backlog is represented by a workload $W_{A+B}^{\phi}$ of a stationary queue of capacity $\phi$ and independent arrival processes $A$ and $B$. Processes $A$ and $B$ can be interpreted as independent demands for a generic resource $\phi$.

Thus, it is of general interest to provide analytical tools for understanding the statistical behavior of $W_{A+B}^{\phi}$. In the context of heavy tails, in [11, theorem 4.4] (see [4,16] for related studies) it was shown that when process $A$ has polynomial characteristics and $B$ is exponentially bounded, then as $x \rightarrow \infty$

$$
\mathbb{P}\left[W_{A+B}^{\phi}>x\right]=\mathbb{P}\left[W_{A}^{\phi-b}>x\right](1+\mathrm{o}(1))
$$

\footnotetext{
* The work of P. Jelenković and P. Momčilović was supported by the NSF PECASE Award No. 9875156.
} 
where $b$ is the mean rate of process $B$ and $W_{A}^{\phi-b}$ is the workload of a queue with reduced load $A$ and capacity $\phi-b$. In [1] the preceding relationship was further investigated and termed reduced load equivalence. For related reduced load equivalence results with polynomial tails see $[3,12,19$, and the references therein].

In this paper we develop a novel large deviation method that under general conditions establishes the reduced load equivalence relationship (1). Our results, in conjunction with the known fact that (1) fails when both processes have lighter than $\mathrm{e}^{-\sqrt{x}}$ characteristics [7], establish the criticality of $\mathrm{e}^{-\sqrt{x}}$ in this setting. Furthermore, the uniform large deviation bound from proposition 1 that plays the central role in our analysis may be of independent interest.

Our main result on reduced load equivalence is stated in theorem 2. The statement of this theorem significantly simplifies when the distribution of deviations in process $B$ has lighter than $\mathrm{e}^{-\sqrt{x}}$ tail; this is presented in proposition 2 . Informally, heuristic of our analysis can be briefly described with the following steps (for large $x$ )

$$
\begin{aligned}
\mathbb{P}\left[W_{A+B}^{1}>x\right] & \approx \mathbb{P}\left[\sup _{t \leqslant l x}\left\{A_{t}-(1-b) t\right\}+\sup _{t \leqslant l x}\left\{B_{t}-b t\right\}>x\right] \\
& \approx \mathbb{P}\left[W_{A}^{1-b}+Z^{+} \sqrt{x}>x\right] \\
& \approx \mathbb{P}\left[W_{A}^{1-b}>x\right],
\end{aligned}
$$

where $Z$ is a Gaussian random variable and $x^{+}$represents the positive part of $x$. The first step is justified by lemma 7. Substantiating approximation (3), i.e., providing a satisfactory bound on the second supremum in (2), represents the main technical difficulty. This is facilitated by [10, theorem 3.2]; strengthened versions of this theorem are presented in lemma 5 and proposition 1. Necessary and sufficient conditions for (4) to hold are provided in theorem 1.

Furthermore, we demonstrate the strength of our new approach by showing that under an equivalent set of conditions the results on independent sampling (see $[2,8]$ ) at subexponential time $T$ hold, i.e., for independent $T$ and $B$ as $x \rightarrow \infty$

$$
\mathbb{P}\left[B_{T}>x\right]=\mathbb{P}[T b>x](1+\mathrm{o}(1)) .
$$

Our results are stated in theorem 3 and proposition 3.

\section{Preliminaries}

\subsection{Gaussian insensitivity}

The purpose of this subsection is to provide a framework for justifying (4) of the introduction. Necessary and sufficient conditions for this to hold are provided when $W_{A}^{1-b}$ is square-root insensitive as in the following definition 1 . The square-root insensitivity appears in $[2,8]$.

Throughout the paper, for any two real functions $f(x)$ and $g(x)$, we use the standard notation $f(x) \sim g(x)$ as $x \rightarrow \infty$ to denote $\lim _{x \rightarrow \infty} f(x) / g(x)=1$. 
Definition 1. Random variable $X$ is called square-root insensitive if

$$
\mathbb{P}[X>x-\sqrt{x}] \sim \mathbb{P}[X>x] \text { as } x \rightarrow \infty .
$$

Remark 1. If $X$ is square-root insensitive then $\mathbb{P}[X>x-k \sqrt{x}] \sim \mathbb{P}[X>x]$ as $x \rightarrow \infty$ for any $k$.

The class of long-tailed random variables is the largest operational class of heavytailed random variables.

Definition 2. A nonnegative random variable $X$ is long-tailed $(X \in \mathcal{L})$ if as $x \rightarrow \infty$

$$
\mathbb{P}[X>x+y] \sim \mathbb{P}[X>x], \quad \forall y \geqslant 0 .
$$
ables.

The following lemma relates long-tailed and square-root insensitive random vari-

Lemma 1. $X$ is square-root insensitive if and only if $\sqrt{X^{+}} \in \mathcal{L}$.

Proof. If $\sqrt{X^{+}} \in \mathcal{L}$ then for $x>1$

$$
\begin{aligned}
1 \leqslant \frac{\mathbb{P}[X>x-\sqrt{x}]}{\mathbb{P}[X>x]} & \leqslant \frac{\mathbb{P}\left[\sqrt{X^{+}}>\sqrt{x-\sqrt{x}}\right]}{\mathbb{P}\left[\sqrt{X^{+}}>\sqrt{x}\right]} \\
& \leqslant \frac{\mathbb{P}\left[\sqrt{X^{+}}>\sqrt{x}-1\right]}{\mathbb{P}\left[\sqrt{X^{+}}>\sqrt{x}\right]} \rightarrow 1,
\end{aligned}
$$

as $x \rightarrow \infty$. On the other hand, if $X$ is square-root insensitive then for $x>y \geqslant 0$

$$
\begin{aligned}
1 \geqslant \frac{\mathbb{P}\left[\sqrt{X^{+}}>x+y\right]}{\mathbb{P}\left[\sqrt{X^{+}}>x\right]} & \geqslant \frac{\mathbb{P}\left[X>x^{2}+2 y x+y^{2}\right]}{\mathbb{P}\left[X>x^{2}\right]} \\
& \geqslant \frac{\mathbb{P}\left[X>x^{2}+3 y x\right]}{\mathbb{P}\left[X>x^{2}\right]} \rightarrow 1, \quad \text { as } x \rightarrow \infty .
\end{aligned}
$$

In this paper $C$ denotes a sufficiently large positive constant, while $c$ denotes a sufficiently small positive constant. The values of $C$ and $c$ may vary in different places; for example, $C / 2=C, C^{2}=C, C+1=C$, etc.

The hazard function $Q(x)$ of a random variable (r.v.) $X$ is defined as $Q(x) \triangleq$ $-\log \mathbb{P}[X>x]$.

Lemma 2. If $X$ is square-root insensitive then its hazard function $Q(x)=\mathrm{o}(\sqrt{x})$ as $x \rightarrow \infty$.

Proof. Lemma 1 renders $\sqrt{X^{+}} \in \mathcal{L}$ and, thus, the hazard function of $\sqrt{X^{+}}$is $\mathrm{o}(x)$ as $x \rightarrow \infty$ (see [17, remark 2.6]), or equivalently $Q(x)=\mathrm{o}(\sqrt{x})$ as $x \rightarrow \infty$. 
The following theorem represents the main technical result of this subsection. It provides a tool to justify, in section 3, step (4) of the outline in the introduction.

Theorem 1. Let $Z$ be a standard normal random variable. Then $X$ is square-root insensitive if and only if

$$
\mathbb{P}\left[X>x-Z^{+} \sqrt{x}\right] \sim \mathbb{P}[X>x] \text { as } x \rightarrow \infty .
$$

Remark 2. If $X$ is square-root insensitive then $\mathbb{P}\left[X>x-k Z^{+} \sqrt{x}\right] \sim \mathbb{P}[X>x]$ as $x \rightarrow \infty$ for any $k$.

Proof of theorem 1. (Only if part). For $x>k^{3}>1$

$$
\begin{aligned}
\mathbb{P}\left[X>x-Z^{+} \sqrt{x}\right] \leqslant & \mathbb{P}\left[Z^{+}>\sqrt[3]{x}\right]+\mathbb{P}\left[\sqrt{X^{+}} \geqslant \sqrt{x-Z^{+} \sqrt{x}}, Z^{+} \leqslant \sqrt[3]{x}\right] \\
\leqslant & \mathbb{P}\left[Z^{+}>\sqrt[3]{x}\right]+\mathbb{P}\left[\sqrt{X^{+}} \geqslant \sqrt{x}-Z^{+}, Z^{+} \leqslant \sqrt[3]{x}\right] \\
\leqslant & \mathbb{P}\left[Z^{+}>\sqrt[3]{x}\right]+\mathbb{P}\left[\sqrt{X^{+}}>\sqrt{x}-k\right] \\
& +\frac{1}{\sqrt{2 \pi}} \int_{k}^{\sqrt[3]{x}} \mathbb{P}\left[\sqrt{X^{+}}>\sqrt{x}-u\right] \mathrm{e}^{-u^{2} / 2} \mathrm{~d} u .
\end{aligned}
$$

Lemma 1 implies $\sqrt{X^{+}} \in \mathcal{L}$ and from definition 2 it follows that for any $\varepsilon>0$ there exists $k>0$ such that $\mathbb{P}\left[\sqrt{X^{+}}>\sqrt{x}-u\right] \leqslant \mathbb{P}\left[\sqrt{X^{+}}>\sqrt{x}\right] \mathrm{e}^{\varepsilon u}$ for all $\sqrt{x}-u>k$. This, together with (5) and lemma 2, yields

$$
1 \leqslant \lim _{x \rightarrow \infty} \frac{\mathbb{P}\left[X>x-Z^{+} \sqrt{x}\right]}{\mathbb{P}[X>x]} \leqslant 1+\frac{1}{\sqrt{2 \pi}} \int_{k}^{\infty} \mathrm{e}^{\varepsilon u-u^{2} / 2} \mathrm{~d} u .
$$

Letting $k \rightarrow \infty$ completes the first part of the proof.

(If part). Note that

$$
\mathbb{P}\left[X>x-Z^{+} \sqrt{x}\right] \geqslant \mathbb{P}\left[Z^{+}>1\right] \mathbb{P}[X>x-\sqrt{x}]+\mathbb{P}\left[Z^{+} \leqslant 1\right] \mathbb{P}[X>x],
$$

which, in conjunction with the assumption, implies $\mathbb{P}[X>x-\sqrt{x}] \sim \mathbb{P}[X>x]$ as $x \rightarrow \infty$.

\subsection{Large deviation results}

This subsection presents several large deviation bounds that will be used in proving our main results. We use the following two classes of heavy-tailed distributions:

Definition 3 [5]. A nonnegative r.v. $X$ is called subexponential $(X \in \mathcal{S})$ if as $x \rightarrow \infty$

$$
\mathbb{P}\left[X_{1}+X_{2}>x\right] \sim 2 \mathbb{P}[X>x],
$$

where $X_{1}$ and $X_{2}$ are independent copies of $X$. 
Definition 4 [13]. A nonnegative r.v. $X$ (or its hazard function) belongs to class $\mathcal{S C}$ (subexponential concave) if its hazard function $Q(x)$ is eventually concave, such that, as $x \rightarrow \infty$

$$
\frac{Q(x)}{\log x} \rightarrow \infty
$$

and for $x \geqslant x_{0}, \beta x \leqslant u \leqslant x$,

$$
\frac{Q(x)-Q(u)}{Q(x)} \leqslant \alpha \frac{x-u}{x},
$$

where $0<\alpha<1,0<\beta<1$.

Random variables with hazard functions $(\log x)^{\gamma}, \gamma>1$, and $x^{\alpha}, 0<\alpha<1$, belong to $\mathcal{S C}$. The following lemma states two basic properties of r.v.s in $\mathcal{S C}$; see [10] for additional discussion on class $\mathcal{S C}$.

For a nonnegative random variable $X$ with finite mean $\mathbb{E} X$, the residual distribution $F_{\mathrm{r}}$ is defined by $F_{\mathrm{r}}(x)=(\mathbb{E} X)^{-1} \int_{0}^{x} \mathbb{P}[X>u] \mathrm{d} u, x \geqslant 0$. A random variable $X_{\mathrm{r}}$ with distribution $F_{\mathrm{r}}$ is called the residual variable of $X$.

Lemma 3 [10].

(a) If $Q \in \mathcal{S C}$, then $Q(x) \leqslant Q(u)(x / u)^{\alpha}$ for all $x_{0} \leqslant u \leqslant x$.

(b) If $X \in \mathcal{S C}$ then $X, X_{\mathrm{r}} \in \mathcal{S}$.

In the remaining part of this subsection, we assume that $\left\{X, X_{i}, i \geqslant 1\right\}$ is a sequence of independent and identically distributed (i.i.d.) r.v.s. The next three lemmas will be used in section 3 to estimate the deviations of process $B$.

Lemma 4. If $\mathbb{E} \mathrm{e}^{Q(X)}<\infty$ for some $Q \in \mathcal{S C}$, then for any $\phi>\mathbb{E} X$

$$
\lim _{x \rightarrow \infty} \mathrm{e}^{\mathrm{o}(Q(x))} \mathbb{P}\left[\sup _{n \geqslant 1}\left\{\sum_{i=1}^{n} X_{n}-\phi n\right\}>x\right]=0 .
$$

Proof. The lemma follows from stochastic dominance, lemma 3 and Pakes' theorem [14].

Lemma 5. If $\mathbb{E} \mathrm{e}^{Q(X)}<\infty$ for some $Q \in \mathcal{S C}$, then for all $x$ and $u \geqslant 0$

$$
\mathbb{P}\left[\max _{1 \leqslant n \leqslant x}\left\{\sum_{i=1}^{n} X_{i}-n \mathbb{E} X\right\}>u\right] \leqslant C\left(\mathrm{e}^{-c u^{2} / x}+x \mathrm{e}^{-(1 / 2) Q(u)}\right) .
$$

Proof. See the appendix. 
Lemma 6. Let $N_{x}=\max \left\{n: \sum_{i=1}^{n} X_{i}<x\right\}$. If $X$ is nonnegative and $\mathbb{E} X^{2}<\infty$, then there exists $\delta>0$ such that for all $x$ and $0 \leqslant u \leqslant \delta x$

$$
\mathbb{P}\left[N_{x}-\frac{x}{\mathbb{E} X}>u\right] \leqslant C \mathrm{e}^{-c u^{2} / x} .
$$

Proof. See the appendix.

\section{Main results}

In this section we present our main results on reduced load equivalence and independent sampling. As mentioned in the introduction, these results extend the work of [1,2]. It will become clear from our analysis that the two problems are strongly related: the square-root insensitivity plays a central role in both of them.

We assume that $B$ is a regenerative process with $B_{0}=0$. The length of the $n$th regenerative cycle is denoted by $v_{n}>0$. Random variables $\left\{v_{n}\right\}_{n=1}^{\infty}$ are i.i.d. independent of a.s. finite $v_{0}$ and have finite second moment, $\mathbb{E} v_{1}^{2}<\infty$. With each $v_{n}, n \geqslant 1$, we associate random variables $\gamma_{n}$ and $\gamma_{n}^{*}$. If $T_{n}=\sum_{i=0}^{n} v_{i}$ then

$$
\gamma_{n}=B_{T_{n}}-B_{T_{n-1}}, \quad \gamma_{n}^{*}=\sup _{T_{n-1} \leqslant t \leqslant T_{n}} B_{t}-B_{T_{n-1}},
$$

with $\gamma_{0}=B_{\nu_{0}}<\infty$ and $\gamma_{0}^{*}=\sup _{0 \leqslant t \leqslant \nu_{0}} B_{t}<\infty$ a.s. Random variables $\left\{\gamma_{n}\right\}_{n=1}^{\infty}$ and $\left\{\gamma_{n}^{*}\right\}_{n=1}^{\infty}$ are i.i.d., independent of $\gamma_{0}$ and $\gamma_{0}^{*}$, with finite first moments. Note that, by the SLLN, the mean rate $b \triangleq \mathbb{E} \gamma_{1} / \mathbb{E} v_{1}=\lim _{t \rightarrow \infty} B_{t} / t$ a.s.

The proofs of our main results use the following proposition that generalizes theorem 3.2 of [10] to regenerative processes.

Proposition 1. If $\mathbb{E} \mathrm{e}^{Q\left(\gamma_{i}^{*}\right)}<\infty, i=0,1$, for some $Q \in \mathcal{S C}$, then for all $x$ and $u \geqslant 0$

$$
\mathbb{P}\left[\sup _{0 \leqslant t \leqslant x}\left\{B_{t}-b t\right\}>u\right] \leqslant C\left(\mathrm{e}^{-c u^{2} / x}+\mathrm{e}^{-c x}+x \mathrm{e}^{-c Q(u)}\right) .
$$

Proof. Let $N_{x}=\max \left\{n: \sum_{i=1}^{n} v_{i}<x\right\}$. Since for all $t \geqslant 0$

$$
B_{t}-b t \leqslant \gamma_{0}^{*}+\gamma_{N_{t-v_{0}}+1}^{*}+\sum_{i=1}^{N_{t-v_{0}}} \gamma_{i}-b \sum_{i=1}^{N_{t-v_{0}}} v_{i}
$$

one concludes

$$
\begin{aligned}
& \mathbb{P}\left[\sup _{0 \leqslant t \leqslant x}\left\{B_{t}-b t\right\}>u\right] \\
& \quad \leqslant \mathbb{P}\left[\gamma_{0}^{*}>\frac{u}{4}\right]+\mathbb{P}\left[\gamma_{1}^{*}>\frac{u}{4}\right]+\mathbb{P}\left[\max _{1 \leqslant n \leqslant N_{x}} \sum_{i=1}^{n}\left(\gamma_{i}-b v_{i}\right)>\frac{u}{2}\right] .
\end{aligned}
$$


Lemma 6 provides a bound on $N_{x}$ for all $\delta>0$ small enough

$$
\mathbb{P}\left[N_{x}-\frac{x}{\mathbb{E} \nu_{1}}>\delta x\right] \leqslant C \mathrm{e}^{-c x}
$$

and, hence, the third term in (7) can be upper bound as follows

$$
\begin{aligned}
\mathbb{P}\left[\max _{0 \leqslant n \leqslant N_{x}} \sum_{i=1}^{n}\left(\gamma_{i}-b v_{i}\right)>\frac{u}{2}\right] & \leqslant C \mathrm{e}^{-c x}+\mathbb{P}\left[\max _{0 \leqslant n \leqslant\left(\delta+1 / \mathbb{E} v_{1}\right) x} \sum_{i=1}^{n}\left(\gamma_{i}-b v_{i}\right)>\frac{u}{2}\right] \\
& \leqslant C \mathrm{e}^{-c x}+C\left(\mathrm{e}^{-c u^{2} / x}+x \mathrm{e}^{-(1 / 2) Q(u / 2)}\right),
\end{aligned}
$$

where the last inequality is due to lemma 5. Substituting the preceding bound in (7) leads to

$$
\mathbb{P}\left[\sup _{0 \leqslant t \leqslant x}\left\{B_{t}-b t\right\}>u\right] \leqslant C \mathrm{e}^{-Q(u / 4)}+C \mathrm{e}^{-c x}+C\left(\mathrm{e}^{-c u^{2} / x}+x \mathrm{e}^{-(1 / 2) Q(u / 2)}\right)
$$

and the statement follows by lemma 3(a).

\subsection{Reduced load equivalence}

In this subsection we investigate the tail behavior of the stationary workload of a stable queue. The stationary workload $W_{X}^{\phi}$ of a queue with service rate $\phi$ fed by a process $X$ with stationary increments, is known to satisfy

$$
W_{X}^{\phi} \stackrel{\mathrm{d}}{=} \sup _{t \geqslant 0}\left\{X_{t}-\phi t\right\}
$$

where $\stackrel{\mathrm{d}}{=}$ denotes equality in distribution and $X_{t}$ represents the amount of work that arrives to the queue in $(-t, 0)$; throughout the paper $X$ will be considered equal to $A$, $B$ or $A+B$. In the queueing context, a natural assumption on $B$ is that sample paths are a.s. increasing, i.e., in this subsection $\gamma_{i}=\gamma_{i}^{*}$ for $i \geqslant 0$. For convenience, we put $W_{X} \equiv W_{X}^{1}$ and let $a$ denote the mean rate of process $A$.

The following theorem is the first main result of this paper.

Theorem 2. Let $\mathbb{E} \mathrm{e}^{Q\left(\gamma_{i}\right)}<\infty, i=0,1$, for some $Q \in \mathcal{S C}$ and $\mathbb{E} v_{1}^{2}<\infty$. If $W_{A}^{1-b} \in \mathcal{S}$ is square-root insensitive, $\mathbb{P}\left[W_{A}^{1-b}>x\right]=\mathrm{e}^{-o(Q(x))}$ and for some $a<\phi<1-b$

$$
\lim _{k \rightarrow \infty} \varlimsup_{x \rightarrow \infty} \frac{\mathbb{P}\left[W_{A}^{\phi}>k x\right]}{\mathbb{P}\left[W_{A}^{1-b}>x\right]}=0,
$$

then as $x \rightarrow \infty$

$$
\mathbb{P}\left[W_{A+B}>x\right] \sim \mathbb{P}\left[W_{A}^{1-b}>x\right]
$$

When the regenerative increments of $B$ do not have tails heavier than $\mathrm{e}^{-\theta \sqrt{x}}, \theta>0$, the conditions of the preceding theorem can be relaxed. In particular, the assumptions 
$W_{A}^{1-b} \in \mathcal{S}$ and $\mathbb{P}\left[W_{A}^{1-b}>x\right]=\mathrm{e}^{-\mathrm{o}(Q(x))}$ are not needed. Note that the class of distributions $\mathcal{S C}$ was used in bounding deviations of process $B$ rather than process $A$. In the case when the deviations of process $B$ are not heavier of those attributed to the CLT, in view of theorem 1 , the square-root insensitivity condition on $W_{A}^{1-b}$ is essentially necessary; this is formalized in the following proposition.

Proposition 2. Let $\mathbb{E} \mathrm{e}^{\theta \sqrt{\gamma_{i}}}<\infty, i=0,1$, for some $\theta>0$ and $\mathbb{E} v_{1}^{2}<\infty$. If $W_{A}^{1-b}$ is square-root insensitive and for some $a<\phi<1-b$

$$
\lim _{k \rightarrow \infty} \varlimsup_{x \rightarrow \infty} \frac{\mathbb{P}\left[W_{A}^{\phi}>k x\right]}{\mathbb{P}\left[W_{A}^{1-b}>x\right]}=0,
$$

then as $x \rightarrow \infty$

$$
\mathbb{P}\left[W_{A+B}>x\right] \sim \mathbb{P}\left[W_{A}^{1-b}>x\right] .
$$

These results extend [1, propositions 8.2 and 8.3], where $A$ is assumed to be an On-Off process with a specific form of the distribution of On periods and $B$ is exponentially bounded. In particular, [1, proposition 8.3] assumes that the tail of the residual distribution of On periods is of the form $\mathrm{e}^{-\alpha x^{\beta}}$ with $\beta<1 / 3$.

Possible choices of $A$ include, for instance, an On-Off process with subexponential On periods and a Gaussian process exhibiting long-range dependence, cf. [18]. Next, we specialize our result to the case where $A$ is a stationary On-Off process. For the exact construction of such a process see [9]. Let On periods be equal in distribution to $\tau$. Denote by $r$ and $a$ the peak and average rate, respectively.

Corollary 1. Let $\mathbb{E} \mathrm{e}^{Q\left(\gamma_{i}\right)}<\infty, i=0,1$ for some $Q \in \mathcal{S C}$ and $\mathbb{E} v_{1}^{2}<\infty$. If $\tau_{r} \in \mathcal{S}$ is square-root insensitive, $\mathbb{P}\left[\tau_{r}>x\right]=\mathrm{e}^{-o(Q(x))}, r>1-b>a$ and

$$
\lim _{k \rightarrow \infty} \varlimsup_{x \rightarrow \infty} \frac{\mathbb{P}\left[\tau_{r}>k x\right]}{\mathbb{P}\left[\tau_{r}>x\right]}=0,
$$

then as $x \rightarrow \infty$

$$
\mathbb{P}\left[W_{A+B}>x\right] \sim \mathbb{P}\left[W_{A}^{1-b}>x\right] .
$$

Proof. Follows from theorem 2 and the asymptotics for $W_{A}^{1-b}$ (see [11, theorem 4.3]).

Before we turn to the proofs of theorem 2 and proposition 2, we state an additional preliminary result.

Lemma 7. Let $\mathbb{E} \mathrm{e}^{Q\left(\gamma_{i}\right)}<\infty, i=0,1$ for some $Q \in \mathcal{S C}$. If $\mathbb{P}\left[W_{A}^{1-b}>x\right]=\mathrm{e}^{-\mathrm{o}(Q(x))}$ and for some $a<\phi<1-b$

$$
\lim _{l \rightarrow \infty} \varlimsup_{x \rightarrow \infty} \frac{\mathbb{P}\left[W_{A}^{\phi}>l x\right]}{\mathbb{P}\left[W_{A}^{1-b}>x\right]}=0,
$$


then

$$
\lim _{l \rightarrow \infty} \varlimsup_{x \rightarrow \infty} \frac{\mathbb{P}\left[\sup _{t>l x}\left\{A_{t}+B_{t}-t\right\}>x\right]}{\mathbb{P}\left[W_{A}^{1-b}>x\right]}=0 .
$$

Proof. See the appendix.

We are now ready to present proofs of theorem 2 and proposition 2 .

Proof of theorem 2. The proof consists of deriving upper and lower bounds.

Upper bound. Observe that for $l>0$

$$
\mathbb{P}\left[W_{A+B}>x\right] \leqslant \mathbb{P}\left[\sup _{0 \leqslant t \leqslant l x}\left\{A_{t}+B_{t}-t\right\}>x\right]+\mathbb{P}\left[\sup _{t>l x}\left\{A_{t}+B_{t}-t\right\}>x\right] .
$$

The second term is negligible by lemma 7 as $x \rightarrow \infty$ for large $l$. To estimate the first term, we proceed as follows. By using $\sup _{t}\{f(t)+g(t)\} \leqslant \sup _{t} f(t)+\sup _{t} g(t)$ for any two functions $f(x)$ and $g(x)$ one obtains for $k>0$

$$
\begin{aligned}
& \mathbb{P}\left[\sup _{0 \leqslant t \leqslant l x}\left\{A_{t}+B_{t}-t\right\}>x\right] \\
& \quad \leqslant \mathbb{P}\left[\sup _{0 \leqslant t \leqslant l x}\left\{A_{t}-(1-b) t\right\}+\sup _{0 \leqslant t \leqslant l x}\left\{B_{t}-b t\right\}>x\right] \\
& \quad \leqslant \mathbb{P}\left[W_{A}^{1-b}>x-k \sqrt{x}\right]+\mathbb{P}\left[W_{A}^{1-b}+Y_{l x}>x, W_{A}^{1-b} \leqslant x-k \sqrt{x}\right] \\
& \quad \triangleq f_{1}+f_{2},
\end{aligned}
$$

where $Y_{x} \triangleq \sup _{0 \leqslant t \leqslant x}\left\{B_{t}-b t\right\}$. Proposition 1 yields an upper bound on $f_{2}$

$$
\begin{aligned}
f_{2} & \leqslant \int_{0}^{x-k \sqrt{x}} \mathbb{P}\left[Y_{l x}>x-u\right] \mathrm{d} \mathbb{P}\left[W_{A}^{1-b} \leqslant u\right] \\
& \leqslant C \int_{0}^{x-k \sqrt{x}}\left(\mathrm{e}^{-c(x-u)^{2} /(l x)}+\mathrm{e}^{-c l x}+l x \mathrm{e}^{-c Q(x-u)}\right) \mathrm{d} \mathbb{P}\left[W_{A}^{1-b} \leqslant u\right] \\
& \triangleq f_{21}+f_{22}+f_{23} .
\end{aligned}
$$

Integration by parts and change of variables $(z=(x-u) / \sqrt{x})$ result in

$$
\begin{aligned}
f_{21} & \leqslant C \mathrm{e}^{-c x / l}+C l^{-1} \int_{0}^{x-k \sqrt{x}} \frac{x-u}{x} \mathrm{e}^{-c(x-u)^{2} /(l x)} \mathbb{P}\left[W_{A}^{1-b}>u\right] \mathrm{d} u \\
& =C \mathrm{e}^{-c x / l}+C l^{-1} \int_{k}^{\sqrt{x}} z \mathrm{e}^{-c z^{2} / l} \mathbb{P}\left[W_{A}^{1-b}>x-z \sqrt{x}\right] \mathrm{d} z \\
& \leqslant C \mathrm{e}^{-c x / l}+C l^{-1} \mathbb{P}\left[W_{A}^{1-b}+Z^{+} \sqrt{x}>x, Z^{+}>k\right],
\end{aligned}
$$


where r.v. $Z$ is a normal random variable. Combining the preceding bound with lemma 3 and theorem 1 we obtain

$$
\varlimsup_{x \rightarrow \infty} \frac{f_{21}}{\mathbb{P}\left[W_{A}^{1-b}>x\right]} \leqslant C l^{-1} \mathbb{P}\left[Z^{+}>k\right] .
$$

It easily follows that the upper bound for the second term in (10) is $f_{22} \leqslant C \mathrm{e}^{-c l x}$. To handle $f_{23}$, define r.v. $U$ such that $\mathbb{P}[U>x]=\mathrm{e}^{-c Q(x)}$ for $x \geqslant x_{U}$. Then,

$$
\begin{aligned}
f_{23} & \leqslant C l x \mathrm{e}^{-c Q(k \sqrt{x})} \int_{0}^{x-k \sqrt{x}} \mathrm{e}^{-c Q(x-u)} \mathrm{d} \mathbb{P}\left[W_{A}^{1-b} \leqslant u\right] \\
& =C l x \mathrm{e}^{-c Q(k \sqrt{x})} \mathbb{P}\left[U+W_{A}^{1-b}>x, W_{A}^{1-b} \leqslant x-k \sqrt{x}\right] ;
\end{aligned}
$$

thus, by lemma 3(b) and [15, corollary 2] one obtains $f_{23}=\mathrm{o}\left(\mathbb{P}\left[W_{A}^{1-b}>x\right]\right)$ as $x \rightarrow \infty$.

Combining the bounds on $f_{21}, f_{22}$ and $f_{23}$ with (10), (9) and square-root insensitivity of $W_{A}^{1-b}$ results, after passing $k \rightarrow \infty$, in

$$
\varlimsup_{x \rightarrow \infty} \frac{\mathbb{P}\left[\sup _{0 \leqslant t \leqslant l x}\left\{A_{t}+B_{t}-t\right\}>x\right]}{\mathbb{P}\left[W_{A}^{1-b}>x\right]}=1 .
$$

Therefore, the proof of the upper bound is concluded by recalling (8) and lemma 7 .

Lower bound. As usual, the lower bound is somewhat easier:

$$
\begin{aligned}
\mathbb{P}\left[W_{A+B}>x\right] & \geqslant \mathbb{P}\left[\sup _{0 \leqslant t \leqslant l x}\left\{A_{t}+B_{t}-t\right\}>x\right] \\
& \geqslant \mathbb{P}\left[\sup _{0 \leqslant t \leqslant l x}\left\{A_{t}-(1-b) t\right\}-\sup _{0 \leqslant t \leqslant l x}\left\{b t-B_{t}\right\}>x\right] .
\end{aligned}
$$

Hence, for any $k, l>0$,

$$
\mathbb{P}\left[W_{A+B}>x\right] \geqslant \mathbb{P}\left[\sup _{0 \leqslant t \leqslant l x}\left\{A_{t}-(1-b) t\right\}>x+k \sqrt{x}\right] \mathbb{P}\left[\sup _{0 \leqslant t \leqslant l x}\left\{b t-B_{t}\right\} \leqslant k \sqrt{x}\right] .
$$

Note that for $t \geqslant 0$

$$
b t-B_{t} \leqslant b v_{0}+b v_{N_{t-v_{0}+1}}+\sum_{i=1}^{N_{t-v_{0}}}\left(b v_{i}-\gamma_{i}\right)
$$

and, thus, by lemma 6 and the CLT for maximums [6, chapter 7] one obtains

$$
\lim _{k \rightarrow \infty} \varlimsup_{x \rightarrow \infty} \mathbb{P}\left[\sup _{0 \leqslant t \leqslant l x}\left\{b t-B_{t}\right\} \leqslant k \sqrt{x}\right]=1 .
$$

The proof is now completed by dividing both sides of (11) by $\mathbb{P}\left[W_{A}^{1-b}>x+k \sqrt{x}\right]$, letting $x \rightarrow \infty$, using the square-root insensitivity of $W_{A}^{1-b}$, setting first $k \rightarrow \infty$, then $l \rightarrow \infty$ and applying lemma 7 . 
Proof of proposition 2. The proof is identical to the proof of theorem 2 with $Q(x)=$ $\theta \sqrt{x}$ except the derivation of the upper bound for $f_{23}$. In the following we show that $f_{23}=\mathrm{o}\left(\mathbb{P}\left[W_{A}^{1-b}>x\right]\right)$ as $x \rightarrow \infty$. For any $0<\delta<1$ integration by parts yields

$$
\begin{aligned}
f_{23} & \leqslant C l x \int_{0}^{x-k \sqrt{x}} \mathrm{e}^{-c \sqrt{x-u}} \mathrm{~d} \mathbb{P}\left[W_{A}^{1-b} \leqslant u\right] \\
& \leqslant C l x \mathrm{e}^{-c \sqrt{x}}+C l x \int_{\delta x}^{x-k \sqrt{x}} \mathrm{e}^{-c \sqrt{x-u}} \mathrm{~d} \mathbb{P}\left[W_{A}^{1-b} \leqslant u\right] \\
& \leqslant C l x \mathrm{e}^{-c \sqrt{x}}+C l x \int_{\delta x}^{x-k \sqrt{x}} \mathrm{e}^{-c \sqrt{x-u}} \mathbb{P}\left[W_{A}^{1-b}>u\right] \mathrm{d} u .
\end{aligned}
$$

Next, square-root insensitivity yields (see lemma 1) that for any $\varepsilon>0$ there exists $x_{\varepsilon} \geqslant 1$ such that for all $x_{\varepsilon} \leqslant u \leqslant x-k \sqrt{x}$

$$
\frac{\mathbb{P}\left[W_{A}^{1-b}>u\right]}{\mathbb{P}\left[W_{A}^{1-b}>x-k \sqrt{x}\right]} \leqslant C \mathrm{e}^{\varepsilon(\sqrt{x}-\sqrt{u})} .
$$

By using the preceding bound in (12) and noting the concavity of the integrand one obtains for $\delta x \geqslant x_{\varepsilon}$

$$
\begin{aligned}
f_{23} \leqslant & C l x \mathrm{e}^{-c \sqrt{x}}+C l x \mathbb{P}\left[W_{A}^{1-b}>x-k \sqrt{x}\right] \int_{\delta x}^{x-k \sqrt{x}} \mathrm{e}^{-c \sqrt{x-u}+\varepsilon(\sqrt{x}-\sqrt{u})} \mathrm{d} u \\
\leqslant & C l x \mathrm{e}^{-c \sqrt{x}}+C l x^{2} \mathbb{P}\left[W_{A}^{1-b}>x-k \sqrt{x}\right]\left(\mathrm{e}^{-c \sqrt{k \sqrt{x}}+\varepsilon(\sqrt{x}-\sqrt{x-k \sqrt{x}})}\right. \\
& \left.+\mathrm{e}^{-\sqrt{x}(c \sqrt{1-\delta}-\varepsilon(1-\sqrt{\delta}))}\right) .
\end{aligned}
$$

Clearly, we can chose $\varepsilon$ and $\delta$ in the preceding inequality to obtain

$$
f_{23} \leqslant C l x \mathrm{e}^{-c \sqrt{x}}+C l x^{2} \mathbb{P}\left[W_{A}^{1-b}>x-k \sqrt{x}\right] \mathrm{e}^{-c \sqrt[4]{x}},
$$

which by lemma 2 and square-root insensitivity yields $f_{23}=\mathrm{o}\left(\mathbb{P}\left[W_{A}^{1-b}>x\right]\right)$ as $x \rightarrow \infty$.

\subsection{Independent sampling}

In this subsection we illustrate the applicability of the developed methodology by investigating the problem of independent sampling at subexponential times that was recently studied in $[2,8]$. The proofs bellow require that $B$ satisfies the CLT and proposition 1; as a sufficient condition for this we assume that $B$ is regenerative. In general, any process $B$ satisfying the CLT and proposition 1 is admissible, e.g., certain Gaussian processes as considered in [18]. We would like to stress that the $\mathcal{S C}$ framework is used only to estimate the behavior of the sampled process $B$ rather then the sampling time $T$. Note that the deviations of process $B$ are due to the large deviations and CLT effects. When the deviations of $B$ are dominated by those of the CLT, the only condition on $T$ that we require is the square-root insensitivity (see proposition 3 ). This, basically necessary, 
square-root insensitivity condition improves on some of the known results in the literature; this will be further elaborated at the end of this section. When the deviations of $B$ are heavier then those stemming from the CLT, one needs an additional set of conditions on $T$ to ensure that those deviations can be tolerated.

Define the maximum $M_{t}=\sup _{0 \leqslant s \leqslant t} B_{s}$. Note that sample paths of $M_{t}$ are nondecreasing. Since $B_{t}$ has positive drift, heuristically, $B_{t}$ is not expected to be much smaller than $M_{t}$. Our theorem below shows that $M_{T}$ and $B_{T}$ have similar tail behavior. For convenience, let mean rate $b=1$.

Theorem 3. Let $\mathbb{E} \mathrm{e}^{Q\left(\gamma_{i}^{*}\right)}<\infty, i=0,1$ for some $Q \in \mathcal{S C}$ and $\mathbb{E} v_{1}^{2}<\infty$. If $T \in \mathcal{S}$ is square-root insensitive and $\mathbb{P}[T>x]=\mathrm{e}^{-\mathrm{o}(Q(x))}$, then as $x \rightarrow \infty$

$$
\mathbb{P}\left[B_{T}>x\right] \sim \mathbb{P}\left[M_{T}>x\right] \sim \mathbb{P}[T>x] .
$$

Proof. Since $B_{T} \leqslant M_{T}$, it suffices to provide an upper bound for $\mathbb{P}\left[M_{T}>x\right]$ and a lower bound for $\mathbb{P}\left[B_{T}>x\right]$.

Upper bound. Write for $0<\delta<1$

$$
\mathbb{P}\left[M_{T}>x\right] \leqslant \mathbb{P}[T>x-k \sqrt{x}]+\mathbb{P}\left[M_{T}>x, \delta x<T \leqslant x-k \sqrt{x}\right]+\mathbb{P}\left[M_{\delta x}>x\right] .
$$

One needs to show that the last two terms are o $(\mathbb{P}[T>x])$ as $x \rightarrow \infty$. Note that, by proposition 1 and lemma 3 ,

$$
\mathbb{P}\left[M_{\delta x}>x\right] \leqslant C\left(\mathrm{e}^{-c x}+x \mathrm{e}^{-c Q(x)}\right) \leqslant C x \mathrm{e}^{-c Q(x)}=\mathrm{o}(\mathbb{P}[T>x]),
$$

as $x \rightarrow \infty$. To deal with the second term in (13), note that, in view of proposition 1 ,

$$
\begin{aligned}
\mathbb{P} & {\left[M_{T}>x, \delta x<T \leqslant x-k \sqrt{x}\right] } \\
& =\int_{\delta x}^{x-k \sqrt{x}} \mathbb{P}\left[M_{u}>x\right] \mathrm{d} \mathbb{P}[T \leqslant u] \\
& \leqslant \int_{\delta x}^{x-k \sqrt{x}} \mathbb{P}\left[\sup _{0 \leqslant s \leqslant u}\left\{B_{s}-s\right\}>x-u\right] \mathrm{d} \mathbb{P}[T \leqslant u] \\
& \leqslant C \int_{\delta x}^{x-k \sqrt{x}}\left(\mathrm{e}^{-c(x-u)^{2} / x}+\mathrm{e}^{-c \delta x}+x \mathrm{e}^{-c Q(x-u)}\right) \mathrm{d} \mathbb{P}[T \leqslant u] .
\end{aligned}
$$

Now, proceed exactly as in bounding $f_{2}$ in the proof of the upper bound in theorem 2 .

Lower bound. Following the steps of the proof of [2, theorem 3.6] we write

$$
\begin{aligned}
\mathbb{P}\left[B_{T}>x\right] & \geqslant \int_{x+k \sqrt{x}}^{\infty} \mathbb{P}\left[B_{u}>x\right] \mathrm{d} \mathbb{P}[T \leqslant u] \\
& \geqslant \inf _{u>x+k \sqrt{x}} \mathbb{P}\left[B_{u}>x\right] \mathbb{P}[T>x+k \sqrt{x}] .
\end{aligned}
$$


Note that due to the monotonicity of $(x-u) / \sqrt{u}$ in $u$ one obtains for $x>k^{2}$

$$
\begin{aligned}
\inf _{u>x+k \sqrt{x}} \mathbb{P}\left[B_{u}>x\right] & \geqslant \inf _{u>x+k \sqrt{x}} \mathbb{P}\left[\frac{B_{u}-u}{\sqrt{u}}>\frac{-k}{\sqrt{1+k / \sqrt{x}}}\right] \\
& \geqslant \inf _{u>x+k \sqrt{x}} \mathbb{P}\left[\frac{B_{u}-u}{\sqrt{u}}>-\frac{k}{2}\right] .
\end{aligned}
$$

Therefore, the square-root insensitivity results in, for an appropriate $\sigma>0$,

$$
\varliminf_{x \rightarrow \infty} \frac{\mathbb{P}\left[B_{T}>x\right]}{\mathbb{P}[T>x]} \geqslant \Phi\left(\frac{k}{2 \sigma}\right)
$$

where $\Phi(\cdot)$ is the distribution function of the standard normal r.v. Letting $k \rightarrow \infty$ concludes the proof.

Proposition 3. If $\mathbb{E} \mathrm{e}^{\theta \sqrt{\gamma_{i}^{*}}}<\infty, i=0,1$ for some $\theta>0, \mathbb{E} v_{1}^{2}<\infty$ and $T$ is squareroot insensitive, then as $x \rightarrow \infty$

$$
\mathbb{P}\left[B_{T}>x\right] \sim \mathbb{P}\left[M_{T}>x\right] \sim \mathbb{P}[T>x] .
$$

Proof. We follow the same steps as in the proof of theorem 3. The only difference is that a bound on $C \int_{\delta x}^{x-k \sqrt{x}} x \mathrm{e}^{-c \sqrt{x-u}} \mathrm{~d} \mathbb{P}[T \leqslant u]$ is obtained using the same arguments as in bounding $f_{23}$ in the proof of proposition 2 .

The preceding proposition fully generalizes proposition 3.1 of [2] and shows that theorems 3.8, 3.10 and 3.11 of [2] hold under less restrictive conditions. In addition, theorem 3 provides an alternative set of conditions, that may appear easier to verify than those stemming from the extreme value theory used in [2, theorem 3.6], under which the sampling result holds. The case of $B_{t}$ being a counting renewal process has been further examined in [8]. When $B_{t}$ is such a process, the only condition in proposition 3 related to $B_{t}$ is $\mathbb{E} v_{1}^{2}<\infty$. An extension of the sampling theorem to the case when the second moment of $v_{1}$ is infinite can be found in [8, theorem 3.1].

\section{Acknowledgement}

We are grateful to Serguei Foss for suggesting lemma 1, which considerably simplified the proofs of lemma 2 and theorem 1 . 


\section{Appendix}

Proof of lemma 5. It is sufficient only to consider the case $x \leqslant C u^{2}$ since otherwise the lemma holds by the appropriate choice of constants. For $\max \{1 / 2, \beta\}<\gamma<1$ let $Y_{i}=X_{i} \mathbb{1}\left\{X_{i} \leqslant \gamma u\right\}$ and $1 /(\gamma u) \leqslant s \leqslant Q(u) / u$. Then

$$
\begin{aligned}
& \mathbb{P}\left[\max _{1 \leqslant n \leqslant x}\left\{\sum_{i=1}^{n} X_{i}-n \mathbb{E} X\right\}>u\right] \\
& \leqslant \mathbb{P}\left[\max _{1 \leqslant n \leqslant x}\left\{\sum_{i=1}^{n} Y_{i}-n \mathbb{E} Y_{1}\right\}>u\right]+x \mathbb{P}[X>\gamma u] \\
& \leqslant \mathbb{P}\left[\max _{1 \leqslant n \leqslant x} \exp \left\{\sum_{i=1}^{n} s\left(Y_{i}-\mathbb{E} Y_{1}\right)\right\}>\mathrm{e}^{s u}\right]+x \mathbb{P}[X>\gamma u] .
\end{aligned}
$$

Next, note that $\exp \left\{\sum_{i=1}^{n} s\left(Y_{i}-\mathbb{E} Y_{1}\right)\right\}$ is a submartingale. Therefore, applying a submartingale inequality (e.g., see [6, theorem 9.4.1]) in the preceding equation leads to

$$
\begin{aligned}
\mathbb{P}\left[\max _{1 \leqslant n \leqslant x}\left\{\sum_{i=1}^{n} X_{i}-n \mathbb{E} X\right\}>u\right] & \leqslant \mathrm{e}^{-s u}\left(\mathbb{E} \mathrm{e}^{s\left(Y_{1}-\mathbb{E} Y_{1}\right)}\right)^{x}+x \mathbb{P}[X>\gamma u] \\
& \leqslant \mathrm{e}^{-s u-s x \mathbb{E} Y_{1}}\left(\mathbb{E} \mathrm{e}^{s Y_{1}}\right)^{x}+C x \mathrm{e}^{-Q(\gamma u)}
\end{aligned}
$$

the last bound is due to Markov's inequality. By repeating exactly the same steps of the proof of [10, theorem 3.2] one can show that there exists a constant $C^{*}$ such that for all $s$ in the given range $\mathbb{E} \mathrm{e}^{s Y_{1}} \leqslant 1+s \mathbb{E} X+C^{*} s^{2}$, which substituted in (A.1) yields

$$
\mathbb{P}\left[\max _{1 \leqslant n \leqslant x}\left\{\sum_{i=1}^{n} X_{i}-n \mathbb{E} X\right\}>u\right] \leqslant \mathrm{e}^{-s u+s^{2} x C^{*}} \mathrm{e}^{s x\left(\mathbb{E} X-\mathbb{E} Y_{1}\right)}+C x \mathrm{e}^{-Q(\gamma u)} .
$$

Next, $\mathbb{E} X-\mathbb{E} Y_{1} \leqslant \sqrt{\mathbb{E} X^{2} \mathbb{P}[X>\gamma u]}$ by Hölder's inequality and, hence,

$$
\mathrm{e}^{s x\left(\mathbb{E} X-\mathbb{E} Y_{1}\right)} \leqslant \mathrm{e}^{C u^{2} \mathrm{e}^{-(1 / 2) Q(\gamma u)}} \leqslant C .
$$

The rest of the proof is exactly the same as in [10, theorem 3.2]: choose $s=Q(u) / u$ if $x \leqslant u^{2} /\left(2 C^{*} Q(u)\right)$ and $s=u /\left(2 x C^{*}\right)$ otherwise. See the proof in [10] for details.

Proof of lemma 6. The definition of $N_{x}$ and Markov's inequality yield for $s>0$

$$
\mathbb{P}\left[N_{x}-\frac{x}{\mathbb{E} X}>u\right]=\mathbb{P}\left[\sum_{i=1}^{\lfloor u+x / \mathbb{E} X\rfloor} X_{i}<x\right] \leqslant \mathrm{e}^{s x}\left(\mathbb{E} \mathrm{e}^{-s X}\right)^{u+x / \mathbb{E} X-1} .
$$

Next we estimate $\mathbb{E} \mathrm{e}^{-s X}$ as follows

$$
\begin{aligned}
\mathbb{E} \mathrm{e}^{-s X} & =\mathbb{E}\left[\mathrm{e}^{-s X} \mathbb{1}\{s X \leqslant 1\}\right]+\mathbb{E}\left[\mathrm{e}^{-s X} \mathbb{1}\{s X>1\}\right] \\
& \leqslant 1-s \mathbb{E} X+s^{2} \mathbb{E} X^{2}+\mathrm{e}^{-1} \mathbb{P}[s X>1],
\end{aligned}
$$


since $\mathrm{e}^{-x} \leqslant 1-x+x^{2}$ for $0 \leqslant x \leqslant 1$. The preceding inequality leads to

$$
\mathbb{E} \mathrm{e}^{-s X} \leqslant 1-s \mathbb{E} X+s^{2}\left(1+\mathrm{e}^{-1}\right) \mathbb{E} X^{2},
$$

which, substituted in (A.2) for $s=(u / x) \mathbb{E} X /\left(2\left(1+\mathrm{e}^{-1}\right) \mathbb{E} X^{2}\right)$, results in

$$
\begin{aligned}
\mathbb{P}\left[N_{x}-\frac{x}{\mathbb{E} X}>u\right] & \leqslant C \mathrm{e}^{s x} \mathrm{e}^{\left(-s \mathbb{E} X+s^{2}\left(1+\mathrm{e}^{-1}\right) \mathbb{E} X^{2}\right)(u+x / \mathbb{E} X-1)} \\
& \leqslant C \mathrm{e}^{-c u^{2} / x} .
\end{aligned}
$$

Proof of lemma 7. Let $0<3 \delta<1-a-b$. Then

$$
\begin{aligned}
& \mathbb{P}\left[\sup _{t>l x}\left\{A_{t}+B_{t}-t\right\}>x\right] \\
& \quad \leqslant \mathbb{P}\left[A_{l x}+B_{l x}-l x+\sup _{t \geqslant l x}\left\{\left(A_{t}-A_{l x}\right)+\left(B_{t}-B_{l x}\right)-(t-l x)\right\}>x\right] \\
& \quad \leqslant \mathbb{P}\left[A_{l x}+B_{l x}>(1-\delta) l x\right]+\mathbb{P}\left[W_{A+B}>(1+l \delta) x\right] \\
& \quad \leqslant \mathbb{P}\left[W_{A+B}^{1-2 \delta}>\delta l x\right]+\mathbb{P}\left[W_{A+B}>\delta l x\right] \\
& \quad \leqslant 2 \mathbb{P}\left[W_{A+B}^{1-2 \delta}>\delta l x\right] \\
& \quad \leqslant 2 \mathbb{P}\left[W_{A}^{a+\delta}>\frac{\delta l x}{2}\right]+2 \mathbb{P}\left[W_{B}^{1-3 \delta-a}>\frac{\delta l x}{2}\right],
\end{aligned}
$$

where we repeatedly used the fact that for any two functions $f(t), g(t), \sup _{t}\{f(t)+$ $g(t)\} \leqslant \sup _{t} f(t)+\sup _{t} g(t)$. Now, since $1-3 \delta-a>b$, the second term in the preceding equation is $\mathrm{o}\left(\mathbb{P}\left[W_{A}^{1-b}>x\right]\right)$ as $x \rightarrow \infty$ by (6) and lemmas 4 and 3. Hence,

$$
\varlimsup_{x \rightarrow \infty} \frac{\mathbb{P}\left[\sup _{t>l x}\left\{A_{t}+B_{t}-t\right\}>x\right]}{\mathbb{P}\left[W_{A}^{1-b}>x\right]} \leqslant 2 \varlimsup_{x \rightarrow \infty} \frac{\mathbb{P}\left[W_{A}^{a+\delta}>\delta l x / 2\right]}{\mathbb{P}\left[W_{A}^{1-b}>x\right]} ;
$$

passing $l \rightarrow \infty$ and using the assumption yield the statement of the lemma.

\section{References}

[1] R. Agrawal, A. Makowski and $\mathrm{Ph}$. Nain, On a reduced load equivalence for fluid queues under subexponentiality, Queueing Systems 33(1-3) (1999) 5-41.

[2] S. Asmussen, C. Klüppelberg and K. Sigman, Sampling at subexponential times, with queueing applications, Stochastic Process. Appl. 79 (1999) 265-286.

[3] S. Borst, O. Boxma and P. Jelenković, Reduced-load equivalence and induced burstiness in GPS queues with long-tailed traffic flows, Queueing Systems 43(4) (2003) 273-306.

[4] O.J. Boxma, Fluid queues and regular variation, Performance Evaluation 27/28 (1996) 699-712.

[5] V.P. Chistyakov, A theorem on sums of independent positive random variables and its application to branching random processes, Theory Probab. Appl. 9 (1964) 640-648.

[6] K.L. Chung, A Course in Probability Theory, 2nd ed. (Academic Press, New York, 1974).

[7] V. Dumas, Private communication (1998).

[8] S. Foss and D. Korshunov, Sampling at random time with a heavy-tailed distribution, Markov Process. Related Fields 6 (2000) 543-568. 
[9] D. Heath, S. Resnick and G. Samorodnitsky, Heavy tails and long range dependence in on/off processes and associated fluid models, Math. Oper. Res. 23 (1998) 145-165.

[10] P. Jelenković and A. Lazar, Asymptotic results for multiplexing subexponential on-off processes, Adv. in Appl. Probab. 31(2) (1999) 394-421.

[11] P. Jelenković and P. Momčilović, Asymptotic loss probability in a finite buffer fluid queue with hetrogeneous heavy-tailed on-off processes, Ann. Appl. Probab. 13(2) (2003) 567-603.

[12] P. Jelenković and P. Momčilović, Large deviation analysis of subexponential waiting times in a processor sharing queue, Math. Oper. Res. 28(3) (2003) 587-608.

[13] A.V. Nagaev, On a property of sums of independent random variables, Theory Probab. Appl. 22(2) (1977) 326-338.

[14] A.G. Pakes, On the tails of waiting-time distribution, J. Appl. Probab. 12 (1975) 555-564.

[15] E.J.G. Pitman, Subexponential distribution functions, J. Austral. Math. Soc. Ser. A 29 (1980) 337347.

[16] T. Rolski, S. Schlegel and V. Schmidt, Asymptotics of Palm-stationary buffer content distribution in fluid flow queues. Adv. in Appl. Probab. 31(1) (1999) 235-253.

[17] K. Sigman, Appendix: A primer on heavy-tailed distributions, Queueing Systems 33(1-3) (1999) 261-275.

[18] B. Zwart, S. Borst and K. Debicki, Reduced-load equivalence for Gaussian processes, Preprint, CWI (2004).

[19] B. Zwart, S. Borst and M. Mandjes, Exact asymptotics for fluid queues fed by multiple heavy-tailed on-off flows, Ann. Appl. Probab., to appear. 